\title{
Beyond the nuclear shell model
}

David J. Dean

Citation: Physics Today 60, 11, 48 (2007); doi: 10.1063/1.2812123

View online: https://doi.org/10.1063/1.2812123

View Table of Contents: http://physicstoday.scitation.org/toc/pto/60/11

Published by the American Institute of Physics

\section{Articles you may be interested in}

Surface-enhanced Raman scattering

Physics Today 60, 40 (2007); 10.1063/1.2812122

The present status of the nuclear shell model

Physics Today 26, 34 (1973); 10.1063/1.3128095

A beachhead on the island of stability

Physics Today 68, 32 (2015); 10.1063/PT.3.2880

A real-world plan to increase diversity in the geosciences

Physics Today 60, 54 (2007); 10.1063/1.2812124

Shell-Model Applications in Nuclear Spectroscopy

Physics Today 31, 68 (1978); 10.1063/1.2994818

A half century of density functional theory

Physics Today 68, 34 (2015); 10.1063/PT.3.2846

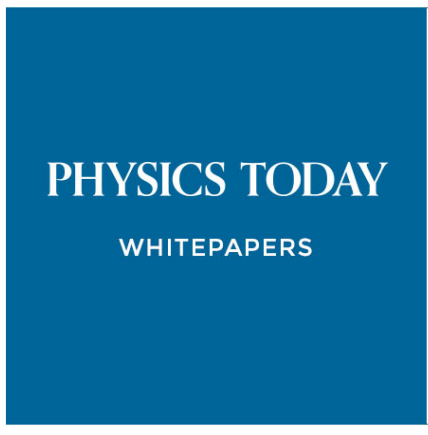

ADVANCED LIGHT CURE ADHESIVES

Take a closer look at what these environmentally friendly adhesive systems can do
READ NOW

PRESENTED BY

Q. MASTERBOND 


\title{
Beyond the nuclear shell model
}

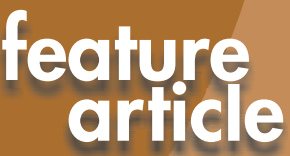

\author{
David J. Dean
}

Nuclear theorists are working to develop a comprehensive understanding of all nuclei. To achieve that goal, they'll need continuing advances in computational power along with data from new and challenging experiments.

David Dean is a Distinguished R\&D Staff Member at Oak Ridge National Laboratory in Oak Ridge, Tennessee.

Nuclei, the fuel that burns in stars, make up $99.9 \%$ of all baryonic matter in the universe. ${ }^{1}$ The complex nature of the nuclear forces among protons and neutrons generates a broad range and diversity of nuclear phenomena. Developing a comprehensive description of nuclei and their reactions represents one of the great intellectual opportunities for physics. As nuclear physicists have seen during the past 10 years, success will require theoretical and experimental investigations of isotopes with unusual neutron-to-proton ratios. Such nuclei, which are typically not found on Earth, are called exotic or rare.

Exotic nuclei, particularly those that have extremely short lifetimes, are difficult to produce experimentally. National user facilities at Argonne and Oak Ridge national laboratories and at Michigan State University, along with other university and national laboratories, are paving the way to exciting initial discoveries. And a new generation of rareisotope facilities is now coming into service to meet the challenge. Notable among them are the Rare Isotope Beam Factory at Japan's RIKEN research institute, which began operation in November 2006, and the Facility for Antiproton and Ion Research, which is under construction at GSI, the heavy-ion research facility in Darmstadt, Germany. Isotope separation techniques continue to be developed as part of the SPIRAL2 project at France's GANIL laboratory and at TRIUMF in Canada. This new generation, along with the proposed Facility for Rare-Isotope Beams (FRIB) in the US, ${ }^{2}$ holds the key to unlocking the mysteries of nuclei and cosmic nuclear production.

Several questions that have come into focus during the past few years illustrate the issues now being addressed in the field of nuclear physics:

- What is the nature of the nuclear force that binds protons and neutrons into stable nuclei and rare isotopes?

- What is the origin of the many simple patterns that emerge in studies of heavy nuclei?

- What is the nature of neutron stars and dense nuclear matter?

-What is the origin of the elements in the cosmos?

- What are the nuclear reactions that drive stars and stellar explosions?

Primary aspects of answering the first two questions are to test the predictive power of models by extending experiments to new regions of mass and neutron-to-proton ratio and to identify new phenomena that challenge existing many-body theory.

On the theoretical side, new and powerful conceptual, analytic, algorithmic, and computational tools are enabling scientists to peer into the inner workings of nuclei with far greater precision than was previously possible. Those tools engender a clear vision to move from a qualitative to a quantitative and comprehensive understanding of all nuclei.

\section{The lay of the land}

How many nuclei are exotic? Figure 1 shows the nuclear landscape as a function of neutron and proton number. Some 270 of the indicated isotopes are stable. About 2000 isotopes have been measured, but a large number-perhaps as many as 4000 - have not been studied in experiment. Unstable, neutron-rich nuclei decay back toward the "valley of stability" via beta decay; proton-rich nuclei approach stability via inverse beta decay. Unstable nuclei can also decay by emitting an alpha particle. The most weakly bound isotopes determine the so-called drip lines; neutron-deficient nuclei lie on the proton drip line, and proton-deficient nuclei on the neutron drip line, whose calculated position varies considerably according to model. Also depicted in the figure is the rapid neutron-capture (r-process) path, which is responsible for the astrophysical production of half of the heavier-than-iron elements in the universe. Scientists know virtually nothing about isotopes along that path.

In 1963 Maria Goeppert Mayer and J. Hans D. Jensen received the Nobel Prize in Physics for their discoveries concerning nuclear shell structure and magic numbers. ${ }^{3}$ Their work led to an extremely successful tool called the shell model. The resulting picture of protons and neutrons (collectively, nucleons) in nuclear shells is analogous to the standard depiction of electrons occupying atomic shells.

In the naive nuclear shell model, nucleons move in an average field, or potential, generated by the whole nucleus; figure 1 includes schematic potential diagrams. Refinements to that simple picture are either theoretically derived or modeled from fits of nucleon interactions in a subset of the shells that are well separated in energy from other shells. When such a subset is filled and the next-higher-energy shell empty, the nucleus is said to have a magic number of protons or neutrons. In the atomic analog, noble gases have magic numbers of electrons. Doubly magic nuclei have magic numbers of both protons and neutrons. The large separation in shell energies at magic numbers means that one could model a nucleus as a core, representing the closed shell, and valence nucleons in the next major shell. That simple picture works well for nuclei near the valley of stability, where the energy needed to liberate a neutron is large.

The bottom right illustration in figure 1 shows the meanfield and shell structure of an exotic nucleus. The last bound 


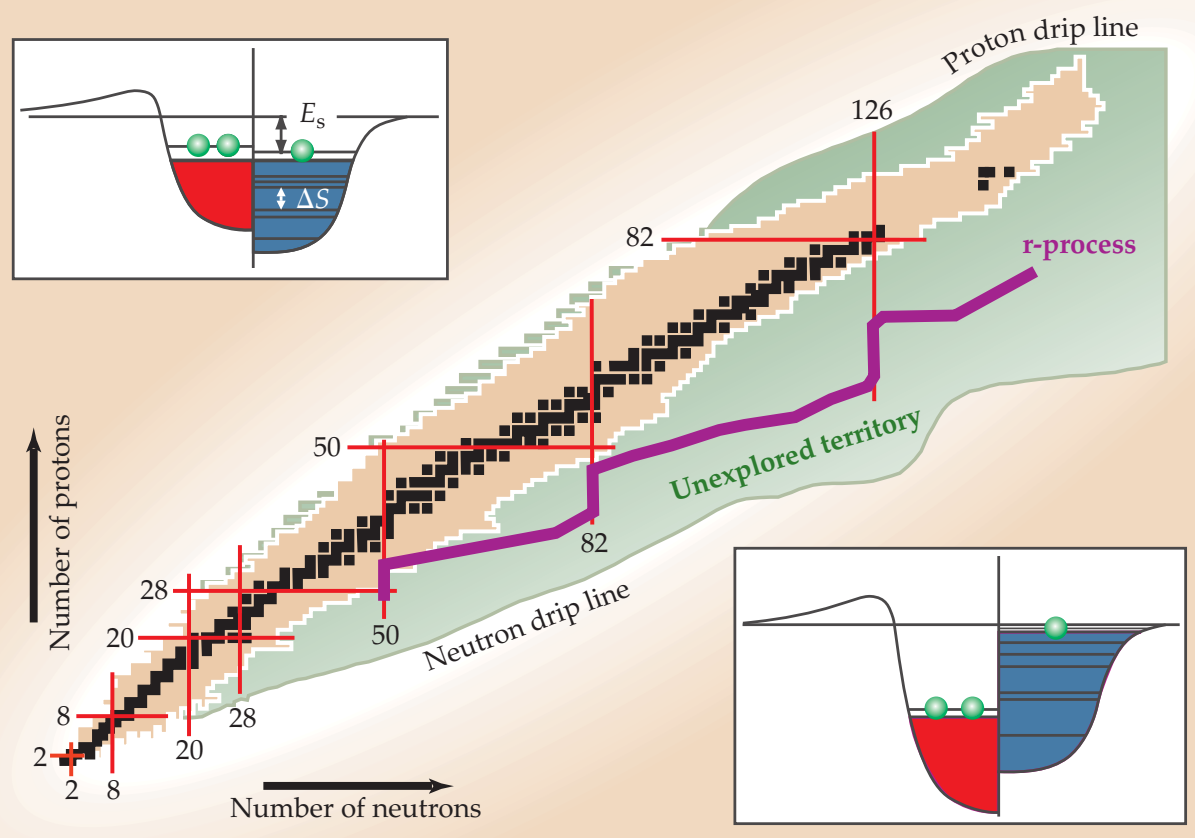

Figure 1. The nuclear landscape depicted here is a chart of nuclides in a plane arranged by neutron and proton number. The black squares represent stable nuclei; the orange region, measured nuclei; and the green region, nuclei that are stable according to calculation but otherwise have not been explored. Red lines indicate magic numbers. The rapid neutroncapture (r-process) path, which describes an important stellar nucleosynthesis chain, lies almost entirely in unexplored territory. The insets show nuclear potentials for protons (red) and neutrons (blue) in a stable nucleus (top) and a neutron-rich weakly bound nucleus (bottom) whose binding or separation energy $E_{\mathrm{s}}$ is rather small. The energy separating major nuclear shells is denoted by $\Delta S$. neutron lies very close in energy to the continuum, and the two most energetic shells are not well separated.

The idea that magic numbers are actually not immutable occurred only during the past 10 years, as experimenters began to probe exotic nuclei. For example, the nucleus beryllium-12 would be expected to exhibit the properties of a nucleus with magic number $N=8$, but as illustrated in figure 2 , the expected large separation in shell energies was not experimentally verified. Similarly, the magic character of neutron number $N=20$ appears to have vanished in the exotic nucleus magnesium-32. It had been widely speculated that doubly magic oxygen-28 would be particularly stable, as is doubly magic ${ }^{16} \mathrm{O}$. Experiments, however, showed that ${ }^{28} \mathrm{O}$ is not even bound. On the other hand, strong indications point to large shell gaps suggestive of magic numbers in other nuclei far from stability: The figure identifies four of those. Three exotic doubly magic nuclei-neutron-deficient tin-100 and neutron-rich nickel-78 and tin-132-are showing surprises as well. For example, the measured half-life of ${ }^{78} \mathrm{Ni}$ is $1 / 3$ to $1 / 4$ as much as theorists predict. ${ }^{4}$ Unstable nuclei near magic numbers may also decay in particularly interesting ways. Researchers have long understood that nuclei along the proton drip line decay by proton emission, and experi- mental evidence now indicates a new and exotic two-proton decay mode $\mathrm{e}^{5}$ in doubly-magic ${ }^{48} \mathrm{Ni}$ or the recently discovered ${ }^{6}$ three-proton decay mode in iron-45.

Experimental observations thus suggest that away from the valley of stability, some aspects of nuclear interactions are amplified in exotic systems and significantly alter nuclear properties. Although the properties of nuclei far from stability may seem a bit esoteric, getting a handle on them could have a profound impact on scientists' understanding of element production in the universe. After all, a number of important nucleosynthesis processes, especially those producing nuclei heavier than carbon and oxygen, occur in nuclei that are very neutron rich or very neutron poor.

Nature does not have the luxury of dealing only with stable nuclei. An understanding of how the elements were and are made depends on an ability to calculate the reaction rates for their production. Those rates, in turn, depend critically on the shell structure of exotic nuclei.

Nuclei far from stability are much more readily influenced by the presence of continuum, resonance, and scattering states than are their stable cousins. The influence is especially pronounced at the neutron drip line. Consider, for example, helium-6 and lithium-11, for which two neutrons

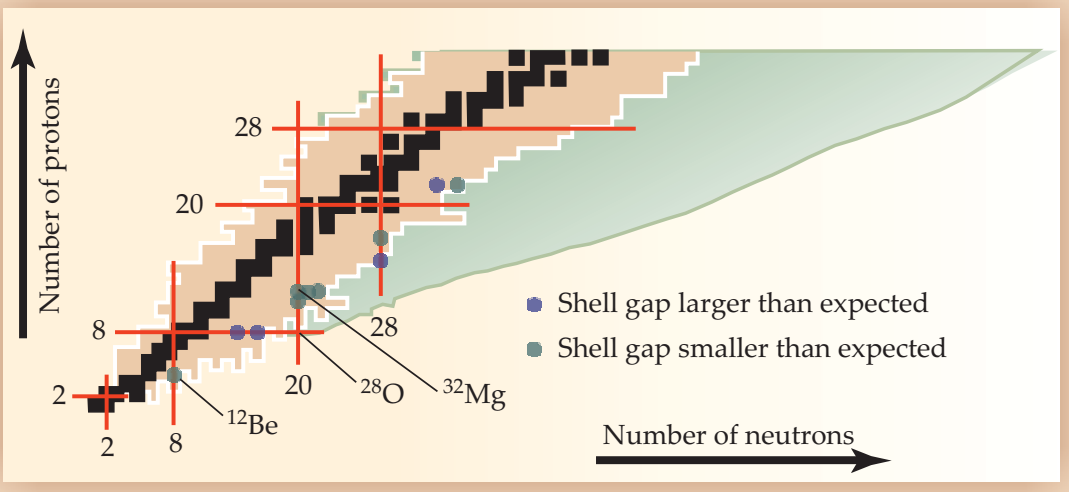

Figure 2. New magic numbers are emerging through accurate measurements of nuclear masses. This enlarged view of the lowmass region of the nuclear landscape indicates several nuclei with unexpected energy gaps between nuclear shells. 
form a halo around a ${ }^{4} \mathrm{He}$ or ${ }^{9} \mathrm{Li}$ core. The halos are indeed extreme. The halo radius of ${ }^{11} \mathrm{Li}$ is similar to the radius of lead-208. Moreover, ${ }^{5} \mathrm{He}$ and ${ }^{10} \mathrm{Li}$ are unbound with respect to nucleon decay and only exist as resonances. It is the addition of two neutrons to the core that causes the binding. Experiments have shown that the two neutrons making up the halo in ${ }^{11} \mathrm{Li}$ influence the charge radius of the ${ }^{9} \mathrm{Li}$ core. ${ }^{7} \mathrm{New}$ experimental facilities will undoubtedly find more surprising halo systems.

Nuclei also serve as laboratories for investigating physics beyond the standard model. For example, neutrinoless double-beta decay may occur in germanium-76. If so, the decay would indicate that the neutrino is its own antiparticle and would help pin down a neutrino mass scale. The neutrino mass scale in turn depends on a "matrix element" that must be calculated accurately, an important challenge for nuclear theory. Another example concerns the search for the dark matter that makes up about $85 \%$ of the material in the universe. One dark-matter candidate is the neutralino, the lightest of the particles predicted in theories that include supersymmetry. If it exists, the neutralino should interact directly with the nuclear spin and may be detected through recoil.

\section{Nuclear interactions}

In her Nobel lecture, Goeppert Mayer stated,

There are essentially two ways in which physicists at present seek to obtain a consistent picture of the atomic nucleus. The first, the basic approach, is to study the elementary particles, their properties and mutual interaction. Thus one hopes to obtain knowledge of the nuclear forces.

If the forces are known, one should in principle be able to calculate deductively the properties of individual nuclei....

The other approach is that of the experimentalist and consists in obtaining by direct experimentation as many data as possible for individual nuclei. One hopes in this way to find regularities and correlations which give a clue to the structure of the nucleus. ...

The shell model, although proposed by theoreticians, really corresponds to the experimentalist's approach. $^{8}$

The theoretical approach requires one to derive forces and solve the quantum many-body problem from them. That differs significantly from using experimental data to fit interactions in given regions of the nuclear landscape, which is and will remain a useful and powerful tool to codify and gain an understanding of nuclear properties in those regions.

Over the past few years, nuclear physics has begun to witness the reemergence of the more basic approach to understanding nuclei. Two avenues may be taken: One involves working from the basic interactions among protons and neutrons, the other is nuclear density functional theory. I will briefly describe both approaches.

Historically, models of the forces between nucleons date back to 1935 and Hideki Yukawa's meson theory. Yukawa posited that pions mediate the force between nucleons, much as photons mediate the electromagnetic force between electrons. However, Yukawa's theory has no real connection to quantum chromodynamics (QCD), which governs the properties of quarks and gluons and is also responsible for the forces that bind nuclei. It was not until the mid-1990s that theoretical groups developed nucleon-nucleon potentials that fit the nucleon-nucleon scattering data to high precision. Those groups were also involved in the first many-body calculations that used the new potentials.

At nearly the same time, a quiet revolution in thinking occurred due to work by Steven Weinberg. In a seminal 1990 paper, Weinberg suggested using techniques of effective field theories - still based on pions and nucleons - that obey the symmetries of QCD. ${ }^{9}$ The strength of an EFT approach to the nuclear force lies in its power-counting capability. That is, the Lagrangian of a theory with nucleons and pions can be expanded order by order in terms of momentum transfer divided by a parameter that sets the momentum scale of the ex- 


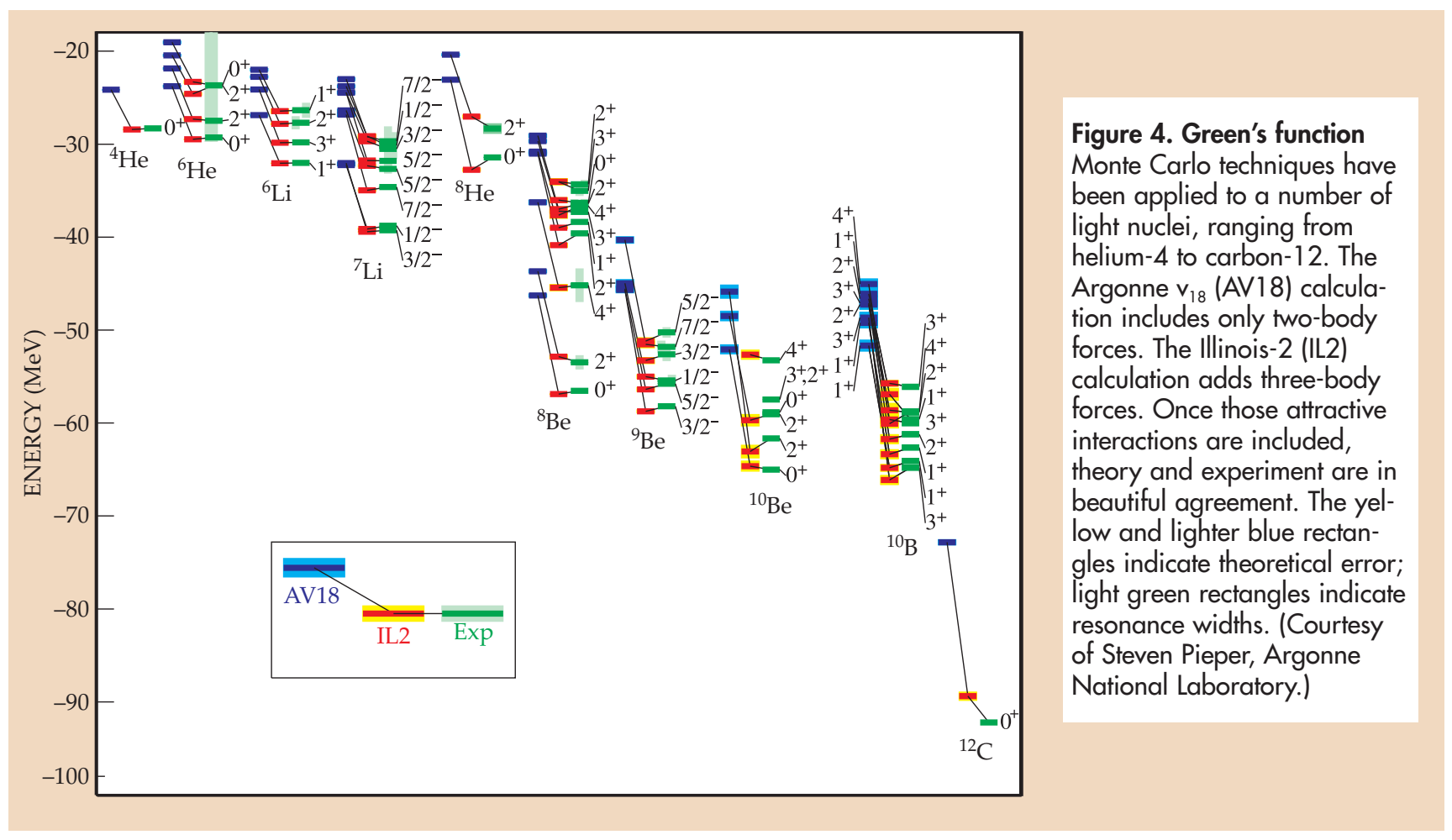

pansion. Usually that parameter is taken as the typical QCD scale of $1 \mathrm{GeV} / c$. The resulting diagrammatic series, analogous to the Feynman diagrams of quantum electrodynamics, has been extended to fourth order. Figure 3 shows how the theory converges toward measured nucleon-nucleon phase shifts. ${ }^{10}$

Another important breakthrough in nuclear theory came from the recognition that at least three-body forces are a part of the story when nucleons are treated as point particles. That realization occurred more than 30 years ago when it was understood that two-nucleon forces alone could not account for the binding energy of the triton (hydrogen-3 nucleus) or alpha particle. At the time, theorists developed several models of three-nucleon forces, but the capability to calculate the effect of three-body forces in anything but the smallest nuclei has developed only in the past 10 years.

Three-body forces are natural inasmuch as nucleons have structure; they are not in fact point particles. The beauty of EFT has been to systematically define the form of those forces, which enter at third order in the expansion. As we shall shortly see, two- and three-body models of the interactions among protons and neutrons enable the calculation of nuclear many-body phenomena.

Effective field theories are a systematic codification of physicists' ignorance about the short range of the strong force. They allow codification of that ignorance, which is captured in contact terms whose coefficients must be determined from experimental nucleon-nucleon scattering data. Some day the exact nature of the force between nucleons may be calculated directly from lattice QCD. ${ }^{11}$ In the meantime significant progress is being made in constructing EFTs that allow one to maintain the symmetries of QCD in the nuclear interactions. That is an important step toward building a comprehensive framework for predictive nuclear theory.

\section{Computing nuclei}

Given a realistic nuclear interaction, the next step is to solve the quantum many-body problem for a specified nucleus with that interaction. The computational effort, however, in- creases exponentially with nucleon number, and the computational challenge is particularly severe when three-nucleon forces become involved. Today, nuclear physics is witnessing breakthrough calculations enabled by terascale $\left(10^{12}\right.$ calculations per second) computation; petascale $\left(10^{15}\right.$ calculations per second) should be available within about three years. Previous generations of scientists could only dream about such computational power.

Several ab initio techniques are now being used to calculate nuclear properties directly. Each of them has advantages and disadvantages. Beginning in the mid-1990s, Joseph Carlson, Vijay Pandharipande, Steven Pieper, and Robert Wiringa vigorously applied Green's function Monte Carlo (GFMC) techniques to light nuclei. ${ }^{12}$ That approach uses Monte Carlo techniques to sample the many-body wavefunction in coordinate, isospin, and spin space for a given interaction. The efforts of Pieper and company marked the beginning of an ab initio understanding of nuclei and the forces binding them and, as illustrated in figure 4 , demonstrate that one can build nuclei from scratch.

A second ab initio approach involves diagonalization of the nuclear Hamiltonian in a finite-dimensional space spanned by a particular basis. The method, pioneered by Bruce Barrett, Petr Navrátil, Erich Ormand, and James Vary, is called the no-core shell model (NCSM). ${ }^{13}$ The method uses realistic interactions projected onto the finite-dimensional space and is approaching the ability to calculate $p$-shell nuclei, such as boron-10, with reasonable accuracy. The method can be thought of as a linear expansion of a correlation operator acting on a reference Slater determinant that is the naive shell model filling.

For both the GFMC and NCSM approaches, computational effort increases exponentially with the number of nucleons. Coupled-cluster methods represent a third approach and are particularly appropriate for closed-shell or subshell nuclei. (A subshell nucleus is analogous to atomic beryllium, which has its $2 s$ electron level filled but its $2 p$ level empty.) An advantage of the method is that its computational 


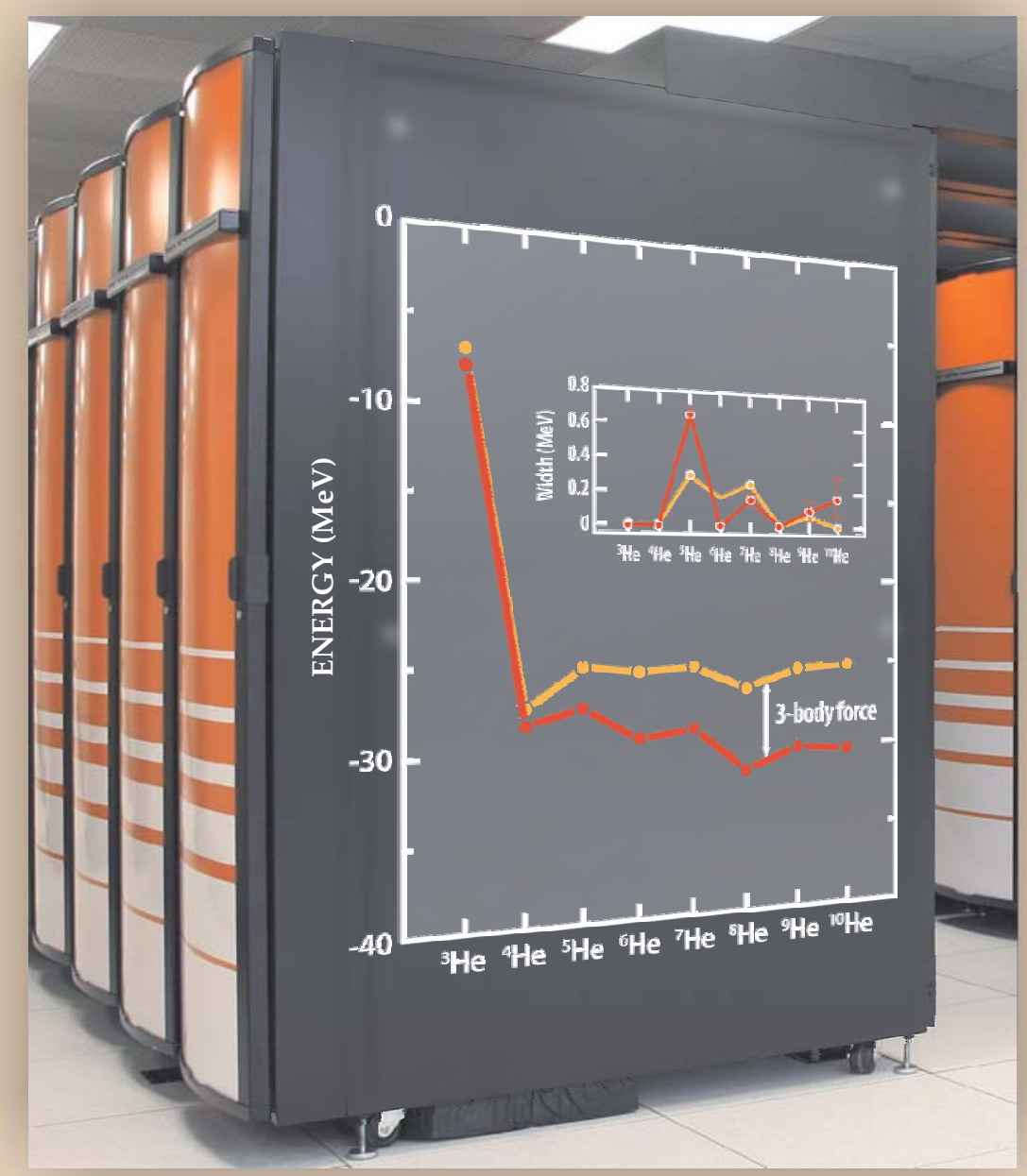

Figure 5. Supercomputers enable nuclear calculations that were impossible only a few years ago. Pictured here is the world's second-fastest computer, Oak Ridge National Laboratory's Jaguar. As an example of its capabilities, the graphs show the first-ever ab initio computation (yellow) of weakly bound nuclei in coupled-cluster theory. The calculation uses Gamow basis states to account for the continuum that is so important for weakly bound and resonant nuclei. The main plot shows the energy of the nuclei in the helium isotopic chain, and the inset gives the width, which is inversely proportional to lifetime. Experimental results are in red. Because the calculations were performed without a three-body force, the calculated energies systematically deviate from experiment.

of particles. And only with that property are applications to larger nuclei and reactions possible. Computationally, coupled-cluster theory requires the solution of coupled nonlinear algebraic equations and fast matrix multiplication.

To explore nuclei far from stability, ab initio techniques will need to be reinvented for continuum problems. Light nuclei with a large neutron excess can develop neutron halos. The old shell-model paradigm of a closed nucleus with harmonic-oscillator valence states simply will not work in such circumstances. The introduction of so-called Gamow basis states enables one to calculate properties for nuclei in which the continuum plays a role. A complex-number version of the coupled-cluster theory was recently developed to calculate widths of states in the helium isotopic chain; that enabled first-principles calculations of energies and lifetimes of unbound states. The calculations, which use only a two-body interaction, are by no means perfect. But as illustrated in figure 5, they do indicate the power of moving beyond the shell model.

Given the dramatic rise in computing power already demonstrated, nuclear physicists should eventually be able to pursue ab initio calculations using coupled-cluster techniques in very massive nuclei. A petascale coupled-cluster calculation would probably involve 100 nucleons in 1000 orbitals; an exascale effort $\left(10^{18}\right.$ calculations per second) would likely involve 200 nucleons in 2000 singleparticle basis states. Such calculations are within the realm of possibility if continued progress yields algorithms that will scale to enormous numbers of processors. Efforts in that direction are under way. Petascale or better computational power should also enable calculations using GFMC, its derivatives, and NCSM into the regions of the nuclear landscape with mass from

requirements increase only polynomially with the number of nucleons.

Coupled-cluster techniques only arrived on the ab initio nuclear scene relatively recently. ${ }^{14}$ Fritz Coester introduced the idea in 1958, and the 1970s saw early work in nuclear physics. ${ }^{15}$ Starting in the late 1970s, quantum chemists began to develop coupled-cluster theory and computations into a rich set of tools, including calculated values of various atomic properties. It is common today for chemists to use coupledcluster theory to correlate up to 100 electrons.

Coupled-cluster theory synthesizes various many-body techniques including cluster expansions, Keith Brueckner's summation of ladder diagrams, the summation of ring diagrams, and an infinite-order generalization of many-body perturbation theory. ${ }^{16}$ The coupled-cluster technique guarantees that energy and errors scale linearly with the number
20 to 40 . The complementary nature of GFMC, NCSM, and coupled-cluster methods makes it important that all three advance so as to benefit from petascale computing and future computational developments.

\section{Density functionals}

Since nuclei are self-bound objects, they produce their own confining potential, or mean field. Density functional theory (DFT) provides the rigorous theoretical foundation for a selfconsistent mean-field description of the nucleus in terms of one-body densities and currents. The idea is to construct a generalized function - a functional - whose input is the proton and neutron densities and currents, and whose output yields the ground-state energy and other properties of the nucleus. The single-particle energies and corresponding oscillator wavefunctions that characterize shell-model calcula- 


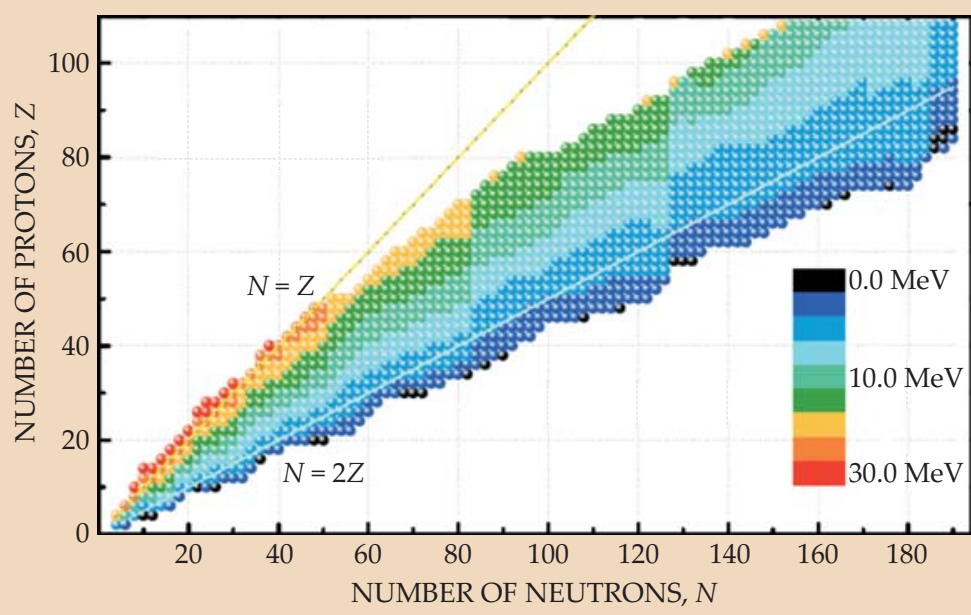

Figure 6. A single energy-density functional predicts the energies needed to separate two neutrons from 1553 different bound nuclei. The results shown here include nuclei with even numbers of both protons and neutrons and allow for nuclear deformations. Clearly visible are neutron shell closures at $N=82,126$, and 184. (Courtesy of Mario Stoitsov, University of Tennessee.)

tions represent a simplified realization of the mean-field idea. Nuclear DFT enables one to describe a broad range of nuclei with various energy-density functionals that include spinand position-dependent correlations. The hope for the longer term is to find a handful of universal functionals - ideally just one-that cover the entire chart of nuclei.

Nuclear DFT efforts usually work quite well in regions where nuclear masses and other properties are experimentally determined. But extrapolations into the unexplored region of neutron-rich nuclei have been problematic. Current and next-generation experimental facilities should enable theorists to obtain a functional parameterization that will describe bulk properties of all nuclei. Theorists will need various nuclear data along lengthy isotopic and isotonic (fixed $N$ ) chains to constrain the isovector part of the energy functional. More specifically, needs include masses, measures of collectivity, and measures of shell evolution for which predictions of currently used functionals diverge. Generally valuable will be data on large deformations at both low and high angular momentum, multipole distributions in neutronrich nuclei, and improved knowledge of the limits of stability in medium-mass neutron-rich nuclei. In particular, data on medium-mass halo candidates at the neutron drip line will provide useful information about the part of the functional influenced by low density and about the gradient corrections in play at the nuclear surface.

The energy-density functional should also lead to a microscopic theory for fission and accurate predictions for reaction cross sections. A more fundamental and accurate description of nuclear reactions would be beneficial to several areas, including basic nuclear physics, science-based stockpile stewardship, and advanced fuel-cycle research for next-generation nuclear reactors. The US Department of Energy's Advanced Fuel Cycle Initiative will require wall-to-wall reactor-core simulations that, among other things, will have an impact on reactor safety and economy. Running those simulations will necessitate advanced nuclear theory calculations of relevant cross sections where experimental data do not exist.

Defining a universal energy-density functional requires computations of ground-state energies and of other observable properties across the nuclear landscape. ${ }^{17}$ Extensive computation time will also be needed, as one must calculate properties of several thousand nuclei several times and allow for nuclear deformation. Such calculations may lead to a functional that can reproduce bulk observables of ab initio many-body calculations in lighter nuclei and reliably predict nuclear properties in regions where no experimental data exist. Figure 6 displays an energy-density functional calculation of the energy needed to separate two neutrons from a given nucleus. It was generated as part of an effort called Building a Universal Nuclear Energy Density Functional, sponsored by DOE's Scientific Discovery through Advanced Computing program.

These are exciting times in nuclear physics. Exotic nuclei are fascinating objects whose properties directly influence element production in the universe and may serve as probes of physics beyond the standard model. Of importance to the broader society, nuclei may be key ingredients in future energy resources and play a significant role in medicine. Theoretical work, coupled with crucial experimental data concerning unstable nuclei, holds the promise of generating a predictive theory and comprehensive understanding of all nuclei.

\section{References}

1. Committee on Nuclear Physics, National Research Council, Nuclear Physics: The Core of Matter, the Fuel of Stars, National Academy Press, Washington, DC (1999), available at http://books.nap.edu/html/nucphys/pdf.html.

2. Rare-Isotope Science Assessment Committee, National Research Council, Scientific Opportunities with a Rare-Isotope Facility in the United States, National Academies Press, Washington, DC (2007), available at http://books.nap.edu/openbook.php?isbn $=0309104084$.

3. M. Goeppert Mayer, Phys. Rev. 74, 235 (1948); O. Haxel, J. H. D. Jensen, H. E. Suess, Phys. Rev. 75, 1766 (1949).

4. P. T. Hosmer et al., Phys. Rev. Lett. 94, 112501 (2005).

5. C. Dossat et al., Phys. Rev. C 72, 054315 (2005).

6. K. Miernik et al., Phys. Rev. C (in press).

7. R. Sánchez et al., Phys. Rev. Lett. 96, $033002(2006)$.

8. M. Goeppert Mayer, in Nobel Lectures, Physics, 1963-1970, Elsevier, Amsterdam (1972), available at http://nobelprize.org/ nobel_prizes/physics/laureates/1963/mayer-lecture.html.

9. S. Weinberg, Phys. Lett. B 251, 288 (1990).

10. D. R. Entem, R. Machleidt, Phys. Rev. C 68, 041001 (2003).

11. N. Ishii, S. Aoki, T. Hatsuda, http://arxiv.org/abs/nucl-th/ 0611096v2.

12. S. C. Pieper, R. B. Wiringa, Annu. Rev. Nucl. Part. Sci. 51, 53 (2001).

13. A. Nogga, P. Navrátil, B. R. Barrett, J. P. Vary, Phys. Rev. C 73, 064002 (2006).

14. M. Włoch et al., Phys. Rev. Lett. 94, 212501 (2005).

15. H. Kümmel, K. H. Lührmann, J. G. Zabolitzky, Phys. Rep. 36, 1 (1978).

16. R. J. Bartlett, M. Musiał, Rev. Mod. Phys. 79, 291 (2007).

17. M. V. Stoitsov et al., Phys. Rev. C 68, 054312 (2003). 